hep-th/9803103

TAUP-2479-98

Imperial/TP/97-98/29

\title{
Supergravity Solutions for Branes Localized Within Branes
}

\author{
N. Itzhaki ${ }^{1}$, A.A. Tseytlin ${ }^{2}$ and S. Yankielowicz ${ }^{1}$ \\ 1 School of Physics and Astronomy, Tel Aviv University, Ramat Aviv, 69978, Israel \\ sanny,shimonya@post.tau.ac.il \\ 2 Blackett Laboratory, Imperial College, London SW7 2BZ, U.K. \\ tseytlin@ic.ac.uk
}

\begin{abstract}
We construct supergravity solutions describing branes (D2-branes or NS 5-branes or waves) localized within D6-branes in the region close to the core of the D6branes. Other similar string-theory and M-theory 'near-core' localized solutions can be found by applying U-duality and/or lifting $D=10$ solutions to $D=11$. In particular, the D2-branes localized on D6-branes is T-dual to a special case of the background describing (D)strings localized on (D)5-branes and thus is also related to a localized intersection of M2-branes and M5-branes. D6+wave configuration is U-dual to a D0-brane localized on a Kaluza-Klein 5-brane or to the fundamental string intersecting a D5-brane with the point of intersection localized on D5-brane.
\end{abstract}

\section{Introduction}

In view of the fundamental role of soliton solutions of supergravity there seems to be little need to justify construction of new such solutions. In particular, recent progress in relating the large $N$ behavior of certain gauge theories to semi-classical supergravity (see, e.g., [1] and references there) serves as a motivation for looking for special solutions describing regions close to the cores of the branes. Also, finding supergravity solutions representing branes ending on branes like Hanany-Witten [2] configuration (and other configurations in different dimensions and with less supersymmetries [3]) should be important.

General solutions representing localized (as opposed to smeared [曰, 5]) intersections of branes or branes ending on branes have not yet been constructed but some progress have 
already being made. The solution describing a string localized on a 5-brane was found (in an implicit form) in [6, 7] (see also [8]). This is a special case of the solution corresponding to a string localized on an intersection [9] of two 5-branes [7, 10]. This latter solution and similar composite solutions involving several branes related by U-duality and lifting to $D=11$ (see also [11]) represent only partially localized intersections, while in the case of, e.g., the Hanany-Witten configuration the localization should be in all relevant dimensions.

In this note we present several explicit solutions for branes which are completely localized within other branes. The $1 / 4$ supersymmetric solutions we find describe, however, only the region close to the core of the 'bigger' brane. Our starting point is the $D=11$ solutions (M2-brane, M5-brane and gravitational wave with $Z_{N}$ identifications in the transverse space) which we shall reduce down to $D=10$ along a circular direction of $S^{3}$ part of the transverse space. The M2-branes solution leads (Sec.2) to $D=10$ background representing D2-branes localized on D6-branes in the region close to the D6-branes core (or, equivalently, it corresponds to the limit of very large charge of D6-brane). The M5branes and waves backgrounds lead (Sec.3) to similar solutions describing the near-core region of D6-branes with, respectively, NS 5-branes and waves localized on it.

Other similar solutions can be found by applying U-duality. In particular, T-dual to our D6+D2 background gives explicit analytic expression for the 'string localized on 5-brane' solution (which was previously found only in the general form parameterized by a function subject to a differential equation [7, 6]) in the special case when the 5-brane is smeared in one of the transverse directions and in the region close to the core of the 5-brane (Sec.4). The system of D1-branes localized within D5-branes is relevant for the $(4,4)$ two-dimensional field theory description of certain five dimensional black holes (see, e.g., [13]). Applying T-duality twice one obtains a system of D0-branes localized within D4-branes related to the DLCQ of the superconformal $(0,2)$ theory [14. Finally, D6+D2 configuration is also related by T-duality to a D-instanton within D3-branes system which corresponds to a localized instanton in the $\mathcal{N}=4$ four-dimensional theory. Therefore, in the spirit of [1], the $\mathcal{N}=44 \mathrm{~d}$ theory on a background of instantons should be dual to type IIB string on a D3+D-instanton background T-dual to the D6+D2 one we find here.

There are several possible generalizations. In particular, one may start with configurations of several intersecting M-branes and reduce along cycle of transverse $S^{3}$ obtaining near-core form of localized intersections involving $n>2$ different D-branes. One example

\footnotetext{
${ }^{1}$ This reduction is a special case of the Hopf reduction discussed in detail in 12 which appeared while this paper was in preparation (the motivation and interpretation of the solutions found in [12] is different from that of the present work).
} 
is provided by the explicit near-core form of solution describing string localized on the intersection of the two 5-branes (Sec.4) which is related [7] to localized $5 \cap 5 \perp 2$ M-theory and $5_{N S} \cap 5_{R} \perp 3$ type IIB theory configurations.

\section{D2-brane within D6-brane}

We would like to find a supergravity solution representing $n_{2}$ D2-branes localized within a collection of $N$ D6-brane in the decoupling (or 'near-core') limit. From the point of view of the field theory on the D6-brane world-volume, it should correspond to localized 2branes 'instantons'. The simplification of considering D6-branes in this limit is that their M-theory counterpart (KK monopole background [15]) becomes an ALE space with an $A_{N-1}$ singularity [16] times a 7-dimensional Minkowski space $M^{(6,1)}$ [17, 18]. This is just a Minkowski space $M^{(10,1)}$ with $Z_{N}$ identifications. Suppose we introduce $n_{2}$ M2-branes along $2+1$ directions in $M^{(6,1)}$. Such M2-branes are invariant under the $Z_{N}$ identifications. Therefore, the corresponding eleven dimensional background will be given by the M2brane solution [19] with the $Z_{N}$ identifications in transverse space. It can then be reduced to ten dimensions to obtain a type IIA solution which, remarkably, can be interpreted as representing the near-core region of a configuration of D2-branes localized within a collection of D6-branes.

Before we turn to the D6+D2 case let us first recall how one can obtain the near-core region of type IIA solution for a collection of $N$ D6-branes upon dimensional reduction of the $A_{N-1}$ space [20]. The metric which corresponds to $M^{(6,1)}$ times an $A_{N-1}$ singularity is

$$
d s_{11}^{2}=d x_{\|}^{2}+d \rho^{2}+\rho^{2}\left(d \tilde{\theta}^{2}+\sin ^{2} \tilde{\theta} d \tilde{\varphi}^{2}+\cos ^{2} \tilde{\theta} d \tilde{\phi}^{2}\right),
$$

where $d x_{\|}^{2}=-d t^{2}+d x_{1}^{2}+\ldots+d x_{6}^{2}, \quad \rho^{2}=x_{7}^{2}+\ldots+x_{10}^{2}, \quad 0 \leq \tilde{\theta} \leq \pi / 2$ and $0 \leq \tilde{\varphi}, \tilde{\phi} \leq 2 \pi$ with the $Z_{N}$ identification $(\tilde{\varphi}, \tilde{\phi}) \sim(\tilde{\varphi}, \tilde{\phi})+(2 \pi / N, 2 \pi / N)$. Defining the new variables

$$
U=\frac{\rho^{2}}{2 N l_{p}^{3}}, \quad \theta=2 \tilde{\theta}, \quad \varphi=\tilde{\varphi}-\tilde{\phi}, \quad \phi=N \tilde{\phi},
$$

we obtain the metric

$$
d s_{11}^{2}=d x_{\|}^{2}+\frac{l_{p}^{3} N}{2 U} d U^{2}+\frac{l_{p}^{3} N U}{2}\left(d \theta^{2}+\sin ^{2} \theta d \varphi^{2}\right)+\frac{2 U l_{p}^{3}}{N}\left[d \phi+\frac{N}{2}(\cos \theta-1) d \varphi\right]^{2},
$$

where $\phi$ has standard period $\phi \sim \phi+2 \pi$. This metric has a Killing vector along the $\phi$ direction and hence it can be reduce to ten dimensions along $x_{11} \equiv R_{11} \phi$. Using the

relation between the eleven dimensional metric and the ten dimensional type IIA string metric, dilaton and the gauge field,

$$
d s_{11}^{2}=e^{4 \phi / 3}\left(d x_{11}+A_{\mu} d x^{\mu}\right)^{2}+e^{-2 \phi / 3} d s_{10}^{2},
$$


we obtain

$$
\begin{aligned}
& d s_{10}^{2}=\alpha^{\prime}\left[\frac{(2 \pi)^{2}}{g_{Y M}} \sqrt{\frac{2 U}{N}} d x_{\|}^{2}+\frac{g_{Y M}}{(2 \pi)^{2}} \sqrt{\frac{N}{2 U}} d U^{2}+\frac{g_{Y M}}{(2 \pi)^{2} \sqrt{2}} \sqrt{N} U^{3 / 2} d \Omega_{2}^{2}\right], \\
& e^{\phi}=\frac{g_{Y M}^{2}}{2 \pi}\left(\frac{2 U}{g_{Y M}^{2} N}\right)^{3 / 4}, \\
& A_{\mu} d x^{\mu}=\frac{N}{2}(\cos \theta-1) d \varphi
\end{aligned}
$$

where $d \Omega_{2}^{2}=d \theta^{2}+\sin ^{2} \theta d \varphi^{2}$. This is the IIA supergravity solution representing a collection of D6-branes 21] in the decoupling limit 22

$$
U=\frac{|x|}{\alpha^{\prime}}=\text { fixed }, \quad g_{Y M}^{2}=(2 \pi)^{4} l_{p}^{3}=(2 \pi)^{4} g_{s} \alpha^{\prime 3 / 2}=\text { fixed }, \quad \alpha^{\prime} \rightarrow 0 .
$$

Let us now consider a configuration of M2-branes stretched along $x_{1}, x_{2}$ directions. For simplicity we shall put all of them at the origin in the transverse space (it is straightforward to generalize the solution to the case where the two-branes are separated along $\left.x_{3}, \ldots, x_{6}\right)$. The required eleven dimensional solution is obvious: it is essentially the same as in [19] but with the above $Z_{N}$ identification of angles of $S^{3}$ (the relevant harmonic function depends only on the radial coordinate $\rho$ via $\rho^{2}+x_{i} x_{i}$ and not on $\tilde{\theta}, \tilde{\phi}$ and $\left.\tilde{\varphi}\right)$. The metric is, therefore,

$$
\begin{gathered}
d s_{11}^{2}=f_{2}^{-2 / 3}\left(-d t^{2}+d x_{1}^{2}+d x_{2}^{2}\right) \\
+f_{2}^{1 / 3}\left[d x_{3}^{2}+\ldots+d x_{6}^{2}+d \rho^{2}+\rho^{2}\left(d \tilde{\theta}^{2}+\sin ^{2} \tilde{\theta} d \tilde{\varphi}^{2}+\cos ^{2} \tilde{\theta} d \tilde{\phi}^{2}\right)\right]
\end{gathered}
$$

where

$$
f_{2}=1+\frac{2^{5} \pi^{2} n_{2} l_{p}^{6}}{\hat{r}^{6}}, \quad \hat{r}^{2}=x_{3}^{2}+\ldots+x_{6}^{2}+\rho^{2}, \quad \rho^{2}=x_{7}^{2}+\ldots+x_{10}^{2},
$$

and the 3-rank tensor has the standard form $C_{012}=f_{2}^{-1}$. Changing the coordinates as in (2) we get

$$
\begin{aligned}
& d s_{11}^{2}=f_{2}^{-2 / 3}\left(-d t^{2}+d x_{1}^{2}+d x_{2}^{2}\right)+f_{2}^{1 / 3}\left(d x_{3}^{2}+\ldots+d x_{6}^{2}+\frac{l_{p}^{3} N}{2 U} d U^{2}\right. \\
& \left.+\frac{l_{p}^{3} N U}{2}\left(d \theta^{2}+\sin ^{2} \theta d \varphi^{2}\right)+\frac{2 U l_{p}^{3}}{N}\left[d \phi+\frac{N}{2}(\cos \theta-1) d \varphi\right]^{2}\right)
\end{aligned}
$$

where now

$$
f_{2}=1+\frac{2^{5} \pi^{2} n_{2} l_{p}^{6}}{\left(x_{3}^{2}+\ldots+x_{6}^{2}+2 N l_{p}^{3} U\right)^{3}} .
$$

As above, we can reduce the solution to ten dimensions along the isometric $\phi$ direction. The result is the type IIA solution describing D2-branes localized within D6-branes (in 
the region near the core of D6-branes)

$$
\begin{aligned}
& d s_{10}^{2}=\alpha^{\prime}\left[f_{2}^{-1 / 2} h_{6}^{-1 / 2}\left(-d t^{2}+d x_{1}^{2}+d x_{2}^{2}\right)+f_{2}^{1 / 2} h_{6}^{-1 / 2}\left(d x_{3}^{2}+\ldots+d x_{6}^{2}\right)\right. \\
& \left.+f_{2}^{1 / 2} h_{6}^{1 / 2}\left(d U^{2}+U^{2} d \Omega_{2}^{2}\right)\right] \\
& e^{\phi}=\frac{g_{Y M}^{2}}{(2 \pi)^{4}} f_{2}^{1 / 4} h_{6}^{-3 / 4} \\
& A_{\mu} d x^{\mu}=\frac{N}{2}(\cos \theta-1) d \varphi
\end{aligned}
$$

where

$$
h_{6}=\frac{g_{Y M}^{2} N}{2(2 \pi)^{4} U},
$$

and $C_{012}=f_{2}^{-1}$. Note that the presence of the D2-branes does not modify the expression for gauge field $A_{\mu}$ and hence the Dirac string singularity can be removed for any integer N

This background has the same 'harmonic function rule' form as the standard $1 / 4$ supersymmetric asymptotically flat D6+D2 bound state solution [5] for which the positions of D2-branes are smeared within the D6-brane directions so that $f_{2}$ and $h_{6}$ are given by

$$
f_{2}=1+\frac{\bar{Q}_{2}}{r}, \quad h_{6}=\alpha^{\prime 2} f_{6}, \quad f_{6}=1+\frac{Q_{6}}{r}, \quad Q_{6}=\frac{1}{2} N g_{s} \alpha^{\prime 1 / 2} .
$$

Here instead we find

$$
f_{2}=1+\frac{Q_{2}}{\left(x_{3}^{2}+\ldots+x_{6}^{2}+4 Q_{6} r\right)^{3}}, \quad h_{6}=\alpha^{\prime 2} \frac{Q_{6}}{r}, \quad r=U / \alpha^{\prime},
$$

i.e. we are restricted to the region close to the D6-brane core (or, equivalently, consider the decoupling limit (6)) but the D2-branes are completely localized within D6-branes ('averaging' over $x_{3}, \ldots, x_{6}$ leads back to the near-core region of the smeared solution). Surprisingly, the D2-brane function $f_{2}$ in (13) 'remembers' its $D=11$ membrane origin: its power of decay with distance is $1 / x^{6}$ compared to $1 / x^{5}$ for the usual D2-brane in ten dimensions. This behavior is characteristic of a string solution, and indeed the above D6+D2 configuration is T-dual to a D-string localized on D5-brane (see Sec.4). Note that the $1 / x^{6}$ is the behavior at the near core region (where our solution is valid). Thus in the full solution the power of decay with distance should interpolates between $1 / x^{6}$ at the near core region and $1 / x^{5}$ far from the horizon.

To summarize, the solution we have found applies only in the decoupling limit. It should be a 'near-core' approximation to a more general solution, yet to be found, describing D2-branes localized within D6-branes and having also the asymptotically flat region (or, equivalently, to the solution with finite $\alpha^{\prime}, g_{s}$ and $r$ ). Trying to add a constant to $h_{6}$ in (13) does not, however, lead to a simple analytic expression for the background (cf. Sec.4). 


\section{$3 \quad$ NS5-brane and wave within D6-brane}

To find a solution describing $n_{5}$ NS5-branes within a collection of $N$ D6-branes we follow the logic of the previous section starting now with the M5-brane solution [23] with the $Z_{N}$ identification in the transverse 3 -sphere. Its metric is

$d s_{11}^{2}=f_{5}^{-1 / 3}\left(-d t^{2}+d x_{1}^{2}+\ldots+d x_{5}^{2}\right)+f_{5}^{2 / 3}\left[d x_{6}^{2}+d \rho^{2}+\rho^{2}\left(d \tilde{\theta}^{2}+\sin ^{2} \tilde{\theta} d \tilde{\varphi}^{2}+\cos ^{2} \tilde{\theta} d \tilde{\phi}^{2}\right)\right]$,

where

$$
f_{5}=1+\frac{\pi n_{5} l_{p}^{3}}{\hat{r}^{3}}, \quad \hat{r}^{2}=x_{6}^{2}+\rho^{2},
$$

and $\rho$ was defined in (7). The change of variables (2) gives

$$
\begin{aligned}
& d s_{11}^{2}=f_{5}^{-1 / 3}\left(-d t^{2}+d x_{1}^{2}+\ldots+d x_{5}^{2}\right)+f_{5}^{2 / 3}\left(d x_{6}^{2}+\frac{l_{p}^{3} N}{2 U} d U^{2}\right. \\
& \left.+\frac{l_{p}^{3} N U}{2}\left(d \theta^{2}+\sin ^{2} \theta d \varphi^{2}\right)+\frac{2 U l_{p}^{3}}{N}\left[d \phi+\frac{N}{2}(\cos \theta-1) d \varphi\right]^{2}\right),
\end{aligned}
$$

where

$$
f_{5}=1+\frac{\pi n_{5} l_{p}^{3}}{\left(x_{6}^{2}+2 N l_{p}^{3} U\right)^{3 / 2}}
$$

Reducing to ten dimensions along $\phi$ we obtain the type IIa solution of $n_{5}$ NS-fivebranes localized within $N$ D6-branes,[]

$$
\begin{aligned}
& d s_{10}^{2}=\alpha^{\prime}\left[h_{6}^{-1 / 2}\left(-d t^{2}+d x_{1}^{2}+\ldots+d x_{5}^{2}\right)+f_{5} h_{6}^{-1 / 2} d x_{6}^{2}+f_{5} h_{6}^{1 / 2}\left(d U^{2}+U^{2} d \Omega_{2}^{2}\right)\right] \\
& e^{\phi}=\frac{g_{Y M}^{2}}{(2 \pi)^{4}} f_{5}^{1 / 2} h_{6}^{-3 / 4} \\
& A_{\mu} d x^{\mu}=\frac{N}{2}(\cos \theta-1) d \varphi
\end{aligned}
$$

where $h_{6}$ was defined in eq.(11). Reducing instead along $x_{5}$ we find a superposition of D4-brane and the near-core region of KK monopole. T-duality in $x_{6}$ and $\phi$ then leads back to D6+ NS5 solution.

Next we turn to waves localized within D6-branes. Starting with the metric of the gravitational wave in $D=11$

$$
\begin{gathered}
d s_{11}^{2}=-d t^{2}+d x_{1}^{2}+f_{0}\left(d t-d x_{1}\right)^{2}+d x_{2}^{2}+\ldots+d x_{6}^{2}+d \rho^{2}+\rho^{2}\left(d \tilde{\theta}^{2}+\sin ^{2} \tilde{\theta} d \tilde{\varphi}^{2}+\cos ^{2} \tilde{\theta} d \tilde{\phi}^{2}\right), \\
f_{0}=\frac{Q_{0}}{\hat{r}^{7}}, \quad \hat{r}^{2}=x_{2}^{2}+\ldots+x_{6}^{2}+\rho^{2},
\end{gathered}
$$

\footnotetext{
${ }^{2}$ This is to be compared with the solution describing delocalised superposition of D6-brane and NS 5 -brane which is a reduction of the 'smeared' superposition of M5-brane and KK monopole given in [5].
} 
and reducing it along $\phi$ as above we find the background representing a wave localized on D6-brane with the metric

$$
d s_{10}^{2}=\alpha^{\prime}\left(h_{6}^{-1 / 2}\left[-d t^{2}+d x_{1}^{2}+f_{0}\left(d t-d x_{1}\right)^{2}+d x_{2}^{2}+\ldots+d x_{6}^{2}\right]+h_{6}^{1 / 2}\left(d U^{2}+U^{2} d \Omega_{2}^{2}\right)\right)
$$

T-duality along the wave $\left(x_{1}\right)$ direction gives a configuration of D5-brane (smeared along $x_{1}$ ) intersected by a fundamental string with the intersection point localized on D5. Sdual to this is NS5-brane intersected by a D-string. T-duality along D-string direction $x_{1}$ gives then a near-core configuration of Kaluza-Klein 5-brane (which is T-dual to NS 5-brane smeared in one transverse direction with T-duality applied in that direction) with D0-brane completely localized on it in all internal coordinates. But this is just the background which is obtained directly by reducing (18) along the $x_{1}$ direction. Again, as usual, the reductions of $D=11$ solutions along different isometric directions give U-dual $D=10$ solutions.

\section{String localized on 5-brane}

Applying T-duality to the localized D6+D2 solution (10) along $x_{2}$ direction one expects to find the D5+D1 solution or S-dual NS5+NS1 solution describing a fundamental string 24] localized on a NS5-brane [25]. Let us show that, indeed, the background T-dual to (10) is a special case of the general 5+1 solution constructed in [6, 7]. The exact string background describing NS $5+1$ configuration is represented by the conformal sigma-model with the following metric, 2 -form and dilaton couplings $(m, n=1,2,3,4)$

$$
\begin{gathered}
d s_{10}^{2}=f_{1}^{-1}(x, y)\left(-d t^{2}+d z^{2}\right)+d y_{n} d y_{n}+f_{5}(x) d x_{m} d x_{m} \\
d B=d f_{1}^{-1} \wedge d t \wedge d z+* d f_{5}, \quad e^{2 \phi}=f_{1}^{-1} f_{5} .
\end{gathered}
$$

Here $\left(z, y_{n}\right)$ are the internal dimensions of the 5 -brane $\left(x_{1}, x_{3}, \ldots, x_{6}\right.$ in (10)), $x_{n}$ are the dimensions transverse to the 5-brane $\left(x_{2}, U, \theta, \varphi\right.$ in (10)) and $f_{5}(x)$ is the harmonic function $\left(\partial^{m} \partial_{m} f_{5} \equiv \partial_{x}^{2} f_{5}=0\right)$ which defines the position of the 5 -brane(s). The string function $f_{1}(x, y)$ must satisfy the condition (Laplace equation in the curved transverse space)

$$
\left[\partial_{x}^{2}+f_{5}(x) \partial_{y}^{2}\right] f_{1}(x, y)=0
$$

Remarkably, this equation admits a simple analytic solution in the special case when (i) the 5-brane is smeared in one of the transverse dimensions (e.g., $x_{2}$ ) so that $f_{5}=$ 
$1+Q_{5} / r, \quad r^{2}=x_{1}^{2}+x_{3}^{2}+x_{4}^{2}$, and (ii) one considers only the near-throat region of the 5-brane where $f_{5} \rightarrow Q_{5} / r$. Assuming that $f_{1}$ depends only on the radial coordinates $r$ and $v, v^{2} \equiv y_{m} y_{m}$, we can put (20) in the form

$$
\left[r^{-1} \partial_{r}\left(r^{2} \partial_{r}\right)+Q_{5} v^{-3} \partial_{v}\left(v^{3} \partial_{v}\right)\right] f_{1}(r, v)=0 .
$$

In terms of the variable $u=2 \sqrt{Q_{5} r}$ it becomes

$$
\left[u^{-3} \partial_{u}\left(u^{3} \partial_{u}\right)+v^{-3} \partial_{v}\left(v^{3} \partial_{v}\right)\right] f_{1}(u, v)=0
$$

i.e. is formally the same as the radial part of the Laplace equation in flat 8 dimensions (with $u^{2}+v^{2}$ as the total distance). This equation is solved, in particular, by

$$
f_{1}=1+\frac{Q_{1}}{\left(v^{2}+u^{2}\right)^{3}}=1+\frac{Q_{1}}{\left(y_{1}^{2}+\ldots+y_{4}^{2}+4 Q_{5} r\right)^{3}},
$$

which has, indeed, the same form as $f_{2}$ in (13). More general solutions are found by separating the string cores in the 5 -brane $y_{n}$ directions. This is in agreement with the possibility to choose a more general M2-brane harmonic function $f_{2}$ in (9) (e.g. a sum of several terms with different centers in $x_{3}, \ldots, x_{6}$ ) and allows, in particular, to construct the solution smeared in these internal directions, thus returning back to $f_{2}$ in (12).

One straightforward generalization is obtained by replacing the product of the flat 4 -space $y_{m}$ and curved 5 -brane 4 -space $x_{n}$ factors in (19) by the direct product of the two 5-brane factors [9]. The resulting exact conformal background describing a string localized on the intersection of the two 5-branes is [6, []

$$
\begin{gathered}
d s_{10}^{2}=f_{1}^{-1}(x, y)\left(-d t^{2}+d z^{2}\right)+f_{5}^{\prime}(y) d y_{n} d y_{n}+f_{5}(x) d x_{m} d x_{m}, \\
d B=d f_{1}^{-1} \wedge d t \wedge d z+* d f_{5}+* d f_{5}^{\prime}, \quad e^{2 \phi}=f_{1}^{-1} f_{5} f_{5}^{\prime},
\end{gathered}
$$

where the harmonic functions $f_{5}(x)$ and $f_{5}^{\prime}(y)\left(\partial_{x}^{2} f_{5}=0, \partial_{y}^{2} f_{5}^{\prime}=0\right)$ define the positions of the two 5 -branes $\left(z, y_{n}\right)$ and $\left(z, x_{m}\right)$ and the string function $f_{1}(x, y)$ satisfies

$$
\left[f_{5}^{\prime}(y) \partial_{x}^{2}+f_{5}(x) \partial_{y}^{2}\right] f_{1}(x, y)=0 .
$$

Assuming that the two 5-branes are smeared in one of the relative transverse directions (e.g., $x_{2}$ and $y_{2}$ ) and considering the near-core region where

$$
f_{5}=\frac{Q_{5}}{r}, \quad f_{5}^{\prime}=\frac{Q_{5}^{\prime}}{r^{\prime}}, \quad r^{2}=x_{1}^{2}+x_{3}^{2}+x_{4}^{2}, \quad r^{\prime 2}=y_{1}^{2}+y_{3}^{2}+y_{4}^{2},
$$

we find that (25) is solved by

$$
f_{1}=1+\frac{Q_{1}}{\left(Q_{5} r+Q_{5}^{\prime} r^{\prime}\right)^{3}}
$$


This gives a simple explicit solution describing a string localized on an intersection of the two 5-branes. Closely related $D=10$ and $D=11$ solutions may be found by using U-duality and lifts to $D=11$ as in [7, 10].

To study some consequences of localization of one brane on another one may consider the action of a D5+D1 probe moving in the background produced by localized D5+D1 source. Equivalent T-dual system is D4+D0 probe moving in D4+D0 source background. Representing the D4+D0 probe by the standard Born-Indeld action with a self-dual gauge field background and ignoring the dependence on internal dimensions of D4-brane probe one finds the following expression for the probe action (see [26])

$$
\begin{gathered}
I_{4+0}=-T_{0} \int d t\left(n_{4}+n_{0}\right. \\
\left.+\left[n_{4} f_{0}^{-1}(x, y)+n_{0} f_{4}^{-1}(x)\right]\left[\sqrt{1-f_{0}(x, y) \dot{y}_{m} \dot{y}_{m}-f_{0}(x, y) f_{4}(x) \dot{x}_{n} \dot{x}_{n}}-1\right]\right) .
\end{gathered}
$$

Here $f_{0}, f_{4}$ are the same as $f_{1}, f_{5}$ in (20) or as $f_{2}, f_{6}$ in (13) (originating from D6+D2, the $\mathrm{D} 4+\mathrm{D} 0$ background is smeared in the two transverse directions) and $n_{0}, n_{4}$ are the charges of the $\mathrm{D} 4+\mathrm{D} 0$ probe. It is obvious that the motion of the probe in both the transverse $\left(x_{n}\right)$ and parallel $\left(y_{m}\right)$ directions to the D4+D0 source depends on the detailed structure of $f_{0}(x, y)$.

\section{Acknowledgments}

The work of N.I. and S.Y. was supported in part by the US-Israel Binational Science Foundation, by GIF - the German-Israeli Foundation for Scientific Research, and by the Israel Science Foundation. The work of A.A.T. was supported by PPARC and the European Commission TMR programme grant ERBFMRX-CT96-0045.

\section{References}

[1] J. Maldacena, The Large N Limit of Superconformal Field Theories and Supergravity, hep-th/9711200.

[2] A. Hanany and E. Witten Type IIB Superstrings, BPS Monopoles, and ThreeDimensional Gauge Dynamics, Nucl. Phys. B492 (1997) 152, hep-th/9611230.

[3] D. Kutasov and A. Giveon, Brane Dynamics and Gauge Theory, hep-th/9802067.

[4] G. Papadopoulos and P.K. Townsend, Intersecting M-branes, Phys. Lett. B380 (1996) 273. 
[5] A.A. Tseytlin, Harmonic superposition of M-branes, Nucl. Phys. B475 (1996) 149, hep-th/9604035.

[6] A.A. Tseytlin, Extremal dyonic black holes in string theory, Mod. Phys. Lett. A11 (1996) 689, hep-th/9601177.

[7] A.A. Tseytlin, Composite BPS configurations of p-branes in 10 and 11 dimensions, Class. Quant. Grav. 14 (1997) 2085, hep-th/9702163.

[8] C.G. Callan, S.S. Gubser, I.R. Klebanov and A.A. Tseytlin, Absorption of fixed scalars and the D-brane approach to black holes, Nucl. Phys. B489 (1997) 65, hepth/9607172; G.T. Horowitz and D. Marolf, Where is the Information Stored in Black Holes?, Phys. Rev. D55 (1997) 3654, hep-th/9610171.

[9] R. Khuri, Remark on string solitons, Phys. Rev. D48 (1993) 2947; J.P. Gauntlett, D.A. Kastor and J. Traschen, Overlapping branes in M-theory, Nucl. Phys. B478 (1996) 544, hep-th/9604179.

[10] J.P. Gauntlett, G.W. Gibbons, G. Papadopoulos and P.K. Townsend, Hyper-Kahler Manifolds and Multiply Intersecting Branes, Nucl. Phys. B500 (1997) 133, hepth/9702202.

[11] J.D. Edelstein, L. Tataru and R. Tatar, Rules for Localized Overlappings and Intersections of $p$-Branes, hep-th/9801049.

[12] M.J. Duff, H. Lü and C.N. Pope, $A d S_{5} \times S^{5}$ Untwisted, hep-th/9803061.

[13] J. Maldacena, Black Holes in String Theory, Ph.D. Thesis, hep-th/9607235.

[14] O. Aharony, M. Berkooz and N. Seiberg, Light-Cone Description of (2,0) Superconformal Theories in Six Dimensions, hep-th/9712117.

[15] P.K. Townsend, Eleven-dimensional supermembrane revisited, Phys. Lett. B350 (1995) 184, hep-th/9501068.

[16] G.W. Gibbons and S.W. Hawking, Gravitational Multi-Instantons, Phys. Lett. 78B (1978) 430.

[17] A. Sen, D0 Branes on $T^{n}$ and Matrix Theory, hep-th/9709220.

[18] N. Seiberg, Why is the Matrix Model Correct?, Phys. Rev. Lett. 79 (1997) 3577.

[19] M.J. Duff and K.S. Stelle, Multimembrane Solutions of D = 11 Supergravity, Phys. Lett. B253 (1991) 113. 
[20] N. Itzhaki, J. Maldacena, J. Sonnenschein and S. Yankielowicz, Supergravity and The Large N Limit of Theories With Sixteen Supercharges, hep-th/9802042.

[21] G. Horowitz and A. Strominger, Black strings and p-branes, Nucl. Phys B360 (1991) 197.

[22] J. Maldacena, Branes probing black holes, hep-th/9709099.

[23] R. Güven, Black p-brane solutions of D=11 supergravity theory, Phys. Lett. B276 (1992) 49.

[24] A. Dabholkar, G.W. Gibbons, J. Harvey and F. Ruiz Ruiz, Superstrings and solitons, Nucl. Phys. B340 (1990) 33.

[25] C.G. Callan, J.A. Harvey and A. Strominger, World sheet approach to heterotic instantons and solitons, Nucl. Phys. B359 (1991) 611.

[26] I. Chepelev and A.A. Tseytlin, Long-distance interactions of D-brane bound states and longitudinal 5-brane in M(atrix) theory, Phys. Rev. D56 (1997) 3672, hepth/9704127. 